\title{
Les droits et devoirs des ministres du culte devant la Cour européenne des droits de l'homme
}

\section{Françoise Curtit}

\section{Q OpenEdition}

12 Journals

Édition électronique

URL : http://journals.openedition.org/rdr/430

DOI : $10.4000 /$ rdr.430

ISSN : 2534-7462

Éditeur

Presses universitaires de Strasbourg

\section{Édition imprimée}

Date de publication : 14 novembre 2019

Pagination : $59-74$

ISBN : 979-10-344-0054-6

ISSN : 2493-8637

\section{Référence électronique}

Françoise Curtit, «Les droits et devoirs des ministres du culte devant la Cour européenne des droits de l'homme », Revue du droit des religions [En ligne], 8 | 2019, mis en ligne le 25 novembre 2019, consulté le 19 novembre 2020. URL : http://journals.openedition.org/rdr/430 ; DOI : https://doi.org/ $10.4000 /$ rdr.430

\section{(c) (†) \$}

La revue du droit des religions est mise à disposition selon les termes de la Creative Commons Attribution - Pas d'Utilisation Commerciale 4.0 International - CC BY-NC 4.0. 


\section{LESDROITSETDEVOIRSDESMINISTRESDU CULTEDEVANTLA COUR EUROPÉENNEDES DROITSDEL'HOMME}

\section{Françoise CURTIT}

Université de Strasbourg / CNRS, Droit, Religion, Entreprise et Société (DRES)

\section{RÉSUMÉ}

Pour la Cour européenne des droits de l'homme, l'autonomie des communautés religieuses constitue un socle sur lequel repose la garantie de la liberté de religion de l'ensemble des fidèles. Ce droit qui leur est reconnu de s'organiser selon leurs propres règles, en étant soumises à un contrôle très restreint des juridictions, leur assure une grande latitude d'action en matière de recrutement, de révocation et d'appréciation du comportement professionnel et personnel de leurs ministres du culte. La mission spécifique de ceux-ci les soumet en outre à un devoir de loyauté accru envers l'organisation religieuse et la doctrine qu'elle professe qui va limiter encore leurs droits et libertés individuels.

\section{Abstract}

According to the European Court of Human Rights, the right to religious autonomy is at the heart of religious freedom for all believers. The right of religious communities to organize themselves according to their own rules, while being subject to limited judicial review, gives them a wide scope for action in the recruitment, dismissal and assessment of the professional and personal conduct of their ministers of religion. Because of their specific mission, the latter are bound by a heightened duty of loyalty towards the religious organization and its doctrine, which further limits their individual rights and freedoms. 
$\mathrm{N}$ i le droit de la Convention européenne des droits de l'homme ni celui de l'Union européenne ne définit ce qu'est ou n'est pas un ministre du culte et ils ont encore moins vocation à déterminer un «statut » au sens d'un ensemble de droits et devoirs applicables à leur situation professionnelle dans ses différents aspects ${ }^{1}$. C'est en effet en premier lieu aux États qu'il revient d'encadrer juridiquement les organisations et activités religieuses et cette primauté leur est reconnue à la fois par l'article $17 \mathrm{du}$ Traité sur le fonctionnement de l'Union européenne et par la jurisprudence de la Cour européenne des droits de l'homme (Cour EDH) qui accorde une large marge nationale d'appréciation en la matière ${ }^{2}$.

La Cour EDH veille cependant au respect par les États des engagements souscrits au titre de la Convention et elle s'est prononcée à plusieurs reprises sur des affaires concernant des personnels d'organisations religieuses, notamment au regard de la protection de la liberté de religion, de la liberté d'association ou de la vie privée. Du côté de l'Union européenne, on ne dispose que de quelques rares décisions, déjà anciennes, par lesquelles la Cour de justice des Communautés européennes a examiné les activités professionnelles de membres de communautés religieuses au regard des dispositions relatives à la réalisation du marché intérieur (liberté de circulation, liberté de prestation de service...) sans faire aucunement référence à la liberté de religion $^{3}$. En 2018, la Cour de justice (CJUE) s'est cependant prononcée dans deux arrêts de Grande Chambre sur une nécessaire mise en balance entre le droit à l'autonomie des Églises et le droit de leurs personnels à ne pas subir de discrimination fondée sur la religion ou les convictions ${ }^{4}$, ces deux

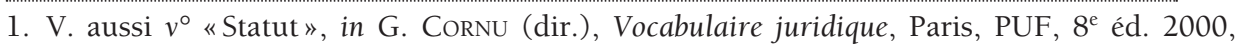
p. 833: «Ensemble cohérent des règles applicables à une catégorie de personnes [...] et qui en déterminent pour l'essentiel la condition et le régime juridique. »

2. V. notamment CEDH, Gde ch., 27 juin 2000, n 27417/95, Cha'are Shalom Ve Tsedek c. France, \& 84: «Eu égard à la marge d'appréciation qu'il faut laisser à chaque État [...] notamment pour ce qui est de l'établissement des délicats rapports entre les Églises et l'État. »

3. V. CJCE, 4 déc. 1974, C-41/74, Yvonne van Duyn c. Home Office: refus d'entrée sur le territoire pour une secrétaire de l'Église de scientologie; 23 oct. 1986, C-300/84, A. J. M. van Roosmalen c. Bestuur van de Bedrijfsvereniging voor de Gezondheid, Geestelijke en Maatschappelijke Belangen : prêtre missionnaire considéré comme un «travailleur non salarié» du point de vue des prestations sociales; 5 oct. 1988, C-196/87, Udo Steymann c. Staatssecretaris van Justitie: activités économiques des membres d'une communauté religieuse.

4. CJUE, Gde ch., 17 avr. 2018, C-414/16, Vera Egenberger c. Evangelisches Werk für Diakonie und Entwicklung: Europe 2018, $\mathrm{n}^{\circ}$ 6, p. 5, note D. SIMON; JSL 2018, n ${ }^{\circ} 454$, p. 4, note M. Hautefort; CJUE, 11 sept. 2018, C-68/17, IR c. JQ: J. Mouly, «Discriminations fondées sur la religion, entreprises de tendance et droit de l'Union. La question du licenciement en raison du remariage après divorce», Droit social 2018, p. 915; X. DelgrangE, "Arrêt "IR": l'entreprise de tendance, de Strasbourg à Luxembourg», JDE 2019/1, p. 17. 
décisions concernant toutefois des employés qui ne peuvent être assimilés à des ministres du culte ${ }^{5}$.

Nous examinerons donc pour l'essentiel la jurisprudence de la Cour EDH relative à des personnes désignées comme «ministres du culte» ou sous diverses dénominations (prêtre, pasteur, rabbin, imam...) utilisées par les confessions elles-mêmes et reprises par les juridictions nationales pour caractériser les personnes en charge des célébrations du culte et de la proclamation du message religieux.

La Cour EDH ne se prononce pas sur le périmètre de la fonction ou sur le contenu de l'activité concernée. Dans une affaire où un membre du Conseil des musulmans du Caucase et de l'université islamique de Bakou voit refuser sa candidature aux élections parlementaires, en vertu de l'incompatibilité prévue par le Code électoral entre un mandat parlementaire et le statut d' " ecclésiastique exerçant une activité professionnelle à caractère religieux », la Cour considère qu'il ne lui appartient pas de «substituer sa propre interprétation à celle des autorités nationales, et notamment des tribunaux». Elle constate simplement que l'absence de définition de ces notions dans la législation de l'Azerbaïdjan laisse aux instances électorales une marge d'appréciation excessive et conclut par conséquent à une violation de l'article $3 \mathrm{du}$ Protocole $\mathrm{n}^{\circ} 1$ (droit à des élections libres) $^{6}$.

De manière générale, quelles que soient l'appellation utilisée et la confession concernée, la Cour EDH reconnaît le rôle central des ministres du culte pour garantir la liberté de religion au sein de communautés, lesquelles vont procéder à leur désignation en fonction de leurs propres règles doctrinales «que les adeptes considèrent souvent comme étant d'origine divine». Elle estime en effet que «Les cérémonies religieuses ont une signification et une valeur sacrée pour les fidèles lorsqu'elles sont célébrées par des ministres du culte qui y sont habilités en vertu de ces règles. La personnalité de ces derniers est assurément importante pour tout membre actif de la communauté, et leur participation à la vie de cette communauté est donc une manifestation particulière de la religion qui jouit en elle-même de la protection de l'article 9 de la Convention ${ }^{7}$ ».

5. En l'occurrence, une candidate à un poste administratif dans une organisation protestante (Vera Engenberger) et un médecin dans un hôpital catholique (JQ).

6. CEDH, 3 déc. 2009, n 37700/05, Seyidzade c. Azerbaïdjan: «clergyman, while engaged in professional religious activity ».

7. CEDH, 8 nov. 2007, n $^{\circ}$ 30273/03, Perry c. Lettonie, § 55. V. aussi CEDH, Gde ch., 26 oct. 2000, n 30985/96, Hassan et Tchaouch c. Bulgarie, § 62. 
La dimension collective de la liberté de religion ici mise en avant se traduit par un droit de libre organisation en lien avec leur doctrine reconnu aux entités religieuses par les États et les juridictions nationales et européenne. Dans la plupart des affaires examinées par la Cour concernant des ministres du culte, leur recrutement, leur révocation et l'appréciation de leur comportement professionnel et personnel s'inscrivent dans ce droit de large autonomie garantie aux entités religieuses (1) qui va alors s'imposer au détriment des droits et libertés des ministres eux-mêmes dont la mission spécifique les soumet à un devoir de loyauté accru envers leur communauté (2).

\section{UN LARGE DROIT À L'AUTONOMIE ACCORDÉ AUX COMMUNAUTÉS RELIGIEUSES}

À plusieurs reprises, la Cour EDH a qualifié l'autonomie des communautés religieuses d'"indispensable au pluralisme dans une société démocratique » et affirmé qu'elle constitue un socle sur lequel repose la garantie de la liberté de religion de l'ensemble des fidèles: "Si l'organisation de la vie de la communauté n'était pas protégée par l'article 9 de la Convention, tous les autres aspects de la liberté de religion de l'individu s'en trouveraient fragilisés ${ }^{8} »$. Ce principe protège notamment les organisations religieuses de toute ingérence injustifiée de l'État, tenu à une obligation de neutralité et d'impartialité qui exclut toute appréciation sur la légitimité des croyances religieuses ou sur ses modalités d'expression ${ }^{9}$.

Garante de la liberté d'exercice du culte, cette autonomie doctrinale a des répercussions importantes sur l'organisation des communautés qui bénéficient ainsi d'une grande liberté de recrutement de leurs personnels (1.1) et en particulier de leurs ministres, lesquels voient leurs garanties procédurales limitées en cas de conflit avec les instances dirigeantes de leur culte (1.2). Même si la Cour reconnaît que leur mission s'inscrit parfois dans le cadre d'une relation de travail, certains droits garantis par la Convention aux salariés ne leur sont pas applicables, tel celui de fonder un syndicat (1.3).

8. CEDH, Gde ch., 26 oct. 2000, n 30985/96, Hassan et Tchaouch c. Bulgarie, § 62; CEDH, 13 déc. 2001, n 45701/99, Église métropolitaine de Bessarabie et autres c. Moldova, § 118.

9. CEDH, Gde ch., 26 oct. 2000, n 30985/96, Hassan et Tchaouch c. Bulgarie, § 78; CEDH, 13 déc. 2001, n 45701/99, Église métropolitaine de Bessarabie et autres c. Moldova, § 117. 


\subsection{UNE GRANDE LIBERTÉ DE RECRUTEMENT DES MINISTRES DU CULTE}

Pour la Cour EDH, le principe d'autonomie des communautés religieuses interdit notamment aux États «d'obliger les communautés religieuses à admettre ou exclure un individu ou à lui confier une responsabilité religieuse quelconque ${ }^{10} »$, conformément à ce qui est d'ailleurs établi dans le droit interne de nombre d'États européens. Dans l'affaire Ahtinen c. Finlande, concernant un pasteur de l'Église évangélique luthérienne qui conteste sa mutation, la Cour note ainsi qu'en droit finlandais l'Église «a le droit d'administrer ses propres affaires. Elle est indépendante dans des matières telles que la nomination de ses prêtres et le service de ces derniers ${ }^{11}$ » et la désignation et la mutation des pasteurs relèvent donc du seul pouvoir du chapitre cathédral, sans intervention possible des pouvoirs publics.

Cette liberté de recrutement largement reconnue ne signifie pas cependant que la communauté religieuse dispose de privilèges dérogatoires lorsqu'il s'agit d'employer des ministres du culte étrangers. Parmi les rares affaires concernant les règles s'appliquant aux ministres musulmans, la Cour a en effet reconnu à plusieurs reprises que l'État pouvait se prévaloir de son droit de refuser l'entrée de son territoire ou un permis de séjour à un individu recruté pour exercer les fonctions d'imam. Dans l'arrêt El Majjaoui c. Pays-Bas, le requérant estime que le refus des autorités néerlandaises de lui délivrer le permis de travail qui lui est indispensable pour être employé comme imam constitue une atteinte injustifiée à son droit à la liberté de religion. La Cour rappelle alors que l'article 9 de la Convention «ne garantit pas aux ressortissants étrangers un droit à obtenir un permis de séjour aux fins d'exercice d'un emploi dans un État contractant, quand bien même l'employeur serait une association religieuse ${ }^{12}{ }$. Dans l'opinion dissidente commune à trois juges, ceux-ci se demandent néanmoins si, au regard de l'article 9, il est acceptable qu'un État, «tenu à un devoir de neutralité relativement à la régulation des groupes religieux», applique à un étranger invité à venir travailler comme ministre religieux les mêmes exigences pour la délivrance d'un permis de travail (salaire minimum légal, absence de main-d'œuvre locale...) qu'aux ressortissants étrangers exerçant d'autres professions. Ils auraient souhaité qu'il

10. CEDH, 14 juin 2007, n 77703/01, Sviato-Mykhaïlivska Parafiya c. Ukraine, \& 146; Gde ch., 9 juill. 2013, n 2330/09, Sindicatul «Păstorul cel Bun»c. Roumanie, § 137; 12 juin 2014, n 56030/07, Fernández Martinez c. Espagne, \& 129.

11. CEDH, 23 sept. 2008, $\mathrm{n}^{\circ}$ 48907/99, Ahtinen c. Finlande, § 41.

12. CEDH, Gde ch., 20 déc. 2007, n² 25525/03, El Majjaoui et Stichting Touba Moskee c. PaysBas, \& 32 [tous les italiques sont de nous]; V. aussi Comm. EDH, déc. 3 déc. 1996, n 32168/96, Нüsnü Öz c. Allemagne. 
soit procédé à un examen de la légitimité des exigences imposées, «alors que dans le choix d'un ministre religieux - qu'il s'agisse d'un pasteur, d'un rabbin ou d'un imam - beaucoup dépend de la question de savoir si la communauté religieuse serait prête à faire confiance à la personne concernée ${ }^{13}{ }$. C'est ici la liberté d'organisation de la communauté qui est mise en avant au détriment de l'application du droit des étrangers retenue par la Grande Chambre, laquelle a finalement prononcé un arrêt de radiation après la suite favorable donnée à une nouvelle demande de permis de travail par le requérant.

Conséquence directe du droit qui leur est reconnu de s'organiser selon leurs règles propres, la liberté de recrutement accordée aux cultes s'accompagne de la même latitude d'action lorsqu'il s'agit de sanctionner ou de révoquer un ministre du culte. Celui-ci bénéficie en effet de garanties procédurales au mieux restreintes lorsqu'il souhaite contester une décision de sa hiérarchie devant les juridictions nationales.

\subsection{UN CONTRÔLE JURIDICTIONNEL RESTREINT DES SANCTIONS DISCIPLINAIRES}

La Cour EDH estime qu'une mesure disciplinaire, voire un licenciement au sein d'une organisation religieuse peuvent être considérés comme ne portant pas atteinte à un droit de l'employé - ou du ministre - reconnu comme défendable en droit interne et se situer donc hors du champ de la protection de l'article 6 de la Convention (droit à un procès équitable). Lorsqu'un droit défendable devant les juridictions internes est néanmoins reconnu, il est alors soumis à un contrôle juridictionnel qui demeure restreint ${ }^{14}$.

La Cour a eu notamment à se prononcer dans deux décisions Baudler et Reuter ${ }^{15}$ à propos de pasteurs employés par les Églises protestantes allemandes qui ont été mis en disponibilité avec une réduction de leurs revenus suite à des dissensions avec leurs paroisses respectives. Les deux ministres ont tenté, sans succès, un recours contre ces décisions devant les instances ecclésiastiques, puis devant les juridictions administratives qui se sont déclarées incompétentes s'agissant de mesures concernant «la mission et le statut»

13. Opinion dissidente commune aux juges Zupančič, Zagrebelski et Myjer.

14. V. G. Gonzalez, «La Convention européenne des droits de l'homme: développement jurisprudentiel d'une conception européenne de la liberté de religion», in F. MESSNER, P.-H. Prélot, J.-M. Woehrling (dir.), Traité de droit français des religions, Paris, LexisNexis, $2^{e}$ éd. 2013, p. 483 et s.; "Consolidation du principe d'autonomie des Églises en droit européen des droits de l'homme», Revue du droit des religions, n 5, 2018, p. 175-180.

15. CEDH, déc. 6 déc. 2011, n 38254/04, Baudler c. Allemagne et, même jour, n 39775/04, Reuter c. Allemagne. 
des pasteurs qui font partie des affaires internes de l'Église. La Cour EDH les a suivies en constatant que les mesures contestées étaient fondées sur les dispositions des Églises organisant le service de leurs pasteurs et donc régies exclusivement par le droit ecclésiastique et non par le droit de l'État, et échappaient par conséquent au contrôle des juridictions étatiques. La Cour conclut que les procédures engagées par les requérants n'ont pas porté sur «un droit que l'on pouvait prétendre, de manière défendable, reconnu en droit allemand», propre à faire appliquer l'article 6 de la Convention.

Dans l'affaire déjà citée concernant la mutation d'un pasteur finlandais, la Cour considère de même que ce type de décision n'est pas susceptible de recours et que rien ne permet de dire que le requérant ait joui d'un «droit» au sens de l'article $6 \S 1$, que ce soit au regard de sa jurisprudence ou du droit national ${ }^{16}$. Elle y évoque d'ailleurs une affaire antérieure à propos de prêtres de l'Église tchèque hussite où elle avait jugé qu'une décision judiciaire sur des questions telles que le maintien en fonction d'un prêtre «serait contraire aux principes d'autonomie et d'indépendance des Églises ${ }^{17} »$.

L'arrêt de Grande Chambre Károly Nagy de $2017^{18}$ confirme cette jurisprudence, à propos d'un pasteur de l'Église réformée de Hongrie révoqué par un tribunal ecclésiastique en raison de déclarations qu'il avait faites dans un journal local. M. Nagy a engagé une action en indemnisation contre l'Église devant le tribunal du travail et devant les juridictions civiles jusqu'à la Cour suprême hongroise, laquelle a jugé que les juridictions de l'État n'étaient pas compétentes du fait que la relation entre le requérant et son employeur relevait du droit ecclésiastique et que la créance patrimoniale n'était dès lors pas susceptible d'exécution par les tribunaux. Pour la Cour de Strasbourg,

«[cette] conclusion des juridictions internes selon laquelle le service pastoral de l'intéressé relevait du droit ecclésiastique et leur décision de mettre fin à la procédure ne sauraient être considérées comme arbitraires ou manifestement déraisonnables [...] Force est pour la Cour de constater que le requérant ne possédait pas un "droit" que l'on pouvait prétendre, au moins de manière défendable, reconnu en droit interne ${ }^{19}$ ».

Cette solution, adoptée par dix voix contre sept, a été critiquée par plusieurs commentateurs qui ont notamment évoqué la construction d'une

16. CEDH, 23 sept. 2008, n 48907/99, Ahtinen c. Finlande, §§ 42-43.

17. Ibid.; V. aussi CEDH, déc. 30 janv. 2001, n 40224/98, Duda et Dudová c. République tchèque.

18. CEDH, Gde ch., 14 sept. 2017, n 56665/09, Károly Nagy c. Hongrie.

19. Ibid., §§ 76-77. 
«immunité de juridiction», en estimant que la nature patrimoniale de la revendication du requérant relative au paiement d'une indemnité aurait dû conduire la Cour à admettre le caractère défendable du grief: «Seule la qualité des parties au litige [pasteur/tribunal ecclésiastique] l'amène à adopter une solution inverse qui tend donc à conférer une immunité de juridiction à tous litiges séculiers entre les membres d'une église ${ }^{20} »$. Il s'agirait même d'une «démission pure et simple du droit européen des droits de l'homme face au droit ecclésiastique, d'autant plus choquante qu'il ne devrait pas suffire qu'une partie à un procès, comportant des aspects patrimoniaux, soit une Église pour qu'à la fois le droit étatique et le droit de la Convention soient mis sur la touche $e^{21} »$. Un avis plus nuancé évoque une «quasi-immunité ecclésiale $^{22} »$ en mentionnant que, dans d'autres affaires, la Cour EDH a reconnu au requérant un droit défendable, objet néanmoins d'un contrôle limité en droit interne.

Ainsi en 2011, à propos de la «mise en indisponibilité» de deux officiers de l'Armée du Salut, dans une «situation comparable à celle des ministres du culte d'autres Églises», la Cour avait suivi les juridictions allemandes en estimant que si les requérants peuvent invoquer un droit reconnu par le droit interne, celui-ci est cependant soumis à un contrôle juridictionnel restreint ${ }^{23}$. La Cour fédérale de justice a en effet créé pour les juridictions de l'État une possibilité, «qui n'existait pas auparavant et qui ne semble d'ailleurs pas être admise par la jurisprudence administrative ni, au demeurant, par la Cour constitutionnelle fédérale», de vérifier la compatibilité d'une mesure ecclésiastique interne avec les principes fondamentaux de l'ordre juridique allemand, lorsqu'il s'agit de litiges dans lesquels la question du statut d'un ecclésiastique n'est appréciée «qu'en tant que question préalable à l'examen du bien-fondé de la demande principale». Selon la Cour fédérale de justice, le droit garanti aux Églises de gérer leurs affaires de manière autonome

20. L. Milano, «Vers une exclusion des litiges entre membres du clergé du champ de l'article 6?, CEDH, Gr. ch, 14 sept. 2017, n 56665/09, Karoly Nagy c. Hongrie», JCP G 2017, act. 1033.

21. J. Mouly, «Discriminations fondées sur la religion, entreprises de tendance et droit de l'Union. La question du licenciement en raison du remariage après divorce», Droit social 2018, p. 919.

22. G. Gonzalez, "Consolidation du principe d'autonomie des Églises en droit européen des droits de l'homme», art. cit., p. 177. V. aussi du même auteur, "L'autonomie ecclésiale dans la jurisprudence de la Cour européenne des droits de l'homme», RDLF 2013, chron. $\mathrm{n}^{\circ} 29$ et «L'autonomie ecclésiale au risque relatif des droits de l'homme», RTDH, $n^{\circ} 2014 / 100$, p. 803-818.

23. CEDH, déc. 6 déc. 2011, n 12986/04, Hanna et Peter Müller c. Allemagne. 
porte alors limitation non pas à l'obligation de l'État de garantir un contrôle judiciaire, mais à l'étendue de celui-ci:

«si la mise en balance du droit d'autonomie des Églises et du droit du plaignant menait à la conclusion que la mesure était exclusivement régie par le droit autonome de l'Église ou de la société religieuse concernée, la conséquence en était que les tribunaux étatiques ne pouvaient pas examiner la légalité (Rechtmässigkeit) de ladite mesure mais uniquement sa validité (Wirksamkeit), c'est-à-dire la question de savoir si elle n'enfreignait pas les principes fondamentaux de l'ordre juridique tels que l'interdiction de l'arbitraire ou les notions de bonnes mœurs et d'ordre public ${ }^{24} »$.

La Cour EDH conclut que les requérants ont eu la possibilité de soulever leurs griefs devant les tribunaux civils, la Cour fédérale de justice ayant considéré que rien ne permettait de dire que la décision de l'Armée du Salut avait été arbitraire ou contraire aux bonnes mœurs ou à l'ordre public. À la différence des affaires Baudler et Reuter, l'article 6 de la Convention trouve donc ici à s'appliquer, mais le grief des requérants tiré du défaut d'accès à un tribunal n'est pas fondé et la requête est donc jugée irrecevable.

Au-delà des questions d'accès aux ou de sortie des fonctions, l'autonomie accordée aux communautés religieuses se traduit également par une limitation des droits et libertés des ministres du culte au cours de leur activité, notamment en matière syndicale.

\subsection{DES LIBERTÉS SYNDICALES RÉDUITES}

S'agissant de l'exercice des libertés syndicales par les ministres du culte, la Grande Chambre a considéré dans l'arrêt Sindicatul "Păstorul cel Bun » ${ }^{25}$ que le refus des autorités roumaines de reconnaître et d'enregistrer un syndicat créé par un groupe de prêtres et d'employés laïcs de l'Église orthodoxe constitue une ingérence de l'État dans l'exercice de la liberté de réunion et d'association (art. $11 \mathrm{CEDH})^{26}$, ingérence qui poursuit cependant un objectif légitime, «à savoir la protection des droits d'autrui, en l'occurrence ceux de

24. Ibid.

25. CEDH, Gde ch., 9 juill. 2013, n 2330/09, Sindicatul «Păstorul cel Bun» c. Roumanie. V. notamment J. Mouly, «L'applicabilité des règles de droit du travail aux membres de l'organisation religieuse », Revue du droit des religions, $n^{\circ}$ 5, 2018, p. 87-104.

26. L'article 11 garantit notamment le droit de fonder un syndicat et d'adhérer à un syndicat de son choix; le droit d'être entendu et «la liberté de défendre les intérêts professionnels des adhérents d'un syndicat par l'action collective de celui-ci, action dont les États 
l'Église orthodoxe roumaine» ( $\$ 158$ ). Elle juge en effet que le refus du tribunal d'enregistrer le syndicat en raison de la non-obtention de l'autorisation de l'archevêque avait été une conséquence directe du droit de la communauté religieuse de s'organiser librement et de fonctionner conformément aux dispositions de son statut (§ 168). L'État «s'est simplement abstenu de s'impliquer dans l'organisation et le fonctionnement autonomes de l'Église orthodoxe roumaine» (§ 166), respectant ainsi son obligation de neutralité. Cet arrêt a été amplement commenté, s'agissant en particulier de savoir si les différents intérêts en présence avaient fait l'objet d'une mise en balance suffisante afin d'apprécier la proportionnalité de l'atteinte portée à la liberté syndicale des requérants ${ }^{27}$.

Il retient aussi notre attention, car la Cour y évoque les différents types de relations juridiques qui lient une organisation cultuelle à ses ministres dans les États européens, afin analyser dans le cas d'espèce la question de l'existence ou non d'une relation de travail (\$ 61). Elle s'appuie pour ce faire sur les critères de la Recommandation $\mathrm{n}^{\circ} 198$ sur la relation de travail de l'OIT, et en premier lieu sur les faits ayant trait à l'exécution du travail et à la rémunération du travailleur. Tout en reconnaissant que «le travail des membres du clergé présente la particularité de poursuivre aussi une finalité spirituelle et d'être accompli dans le cadre d'une Église pouvant prétendre à un certain degré d'autonomie» (§ 144), la Cour se base sur un faisceau d'indices (nomination par l'évêque, supervision de celui-ci, rémunération sur budgets publics, cotisations patronales, prestations sociales...) pour estimer que les membres du clergé de l'Église orthodoxe roumaine accomplissent leur mission dans le cadre d'une relation de travail (§ 148). Celle-ci pourrait entraîner l'applicabilité de certains droits garantis par la Convention aux salariés, dans l'optique de l'arrêt de chambre qui avait estimé que «la relation fondée sur un contrat de travail ne saurait être "cléricalisée" au point d'échapper à toute règle de droit civil ${ }^{28} »$. La Grande Chambre a en effet admis que les prêtres de l'Église orthodoxe roumaine bénéficient de l'applicabilité de l'article 11 de la Convention garantissant la liberté d'association et la liberté syndicale en raison de leur qualité de travailleurs. Rien ne les empêche donc de fonder ou d'adhérer à un syndicat «dont les objectifs

contractants doivent à la fois autoriser et rendre possibles la conduite et le développement»: CEDH, 27 oct. 1975, n 4464/70, Syndicat national de la police belge c. Belgique.

27. V. par ex. R. PIERRE, «La liberté syndicale à l'épreuve du droit d'autonomie des groupements confessionnels», JCP S 2013, 1286. - V. aussi l'opinion en partie dissidente de six des juges.

28. CEDH, 31 janv. 2012, n 2330/09, Sindicatul «Păstorul cel Bun» c. Roumanie, § 65. 
seraient compatibles avec le statut de l'Église et qui ne remettrait pas en question [sa] structure hiérarchique traditionnelle» (§ 170). Mais finalement, on l'a dit, c'est le principe d'autonomie des cultes qui a prévalu en l'espèce au moment de leur reconnaître un droit concret.

Ce droit à l'autonomie reconnu aux communautés religieuses leur garantit de choisir et de gérer leurs personnels conformément à leurs options doctrinales tout en étant soumises à des contrôles très réduits de la part des pouvoirs publics et des juridictions. Il leur permet en outre d'imposer à leurs ministres un devoir de loyauté accru qui va restreindre l'exercice par ceux-ci de leurs droits et libertés individuels.

\section{UN DEVOIR DE LOYAUTÉ ACCRU IMPOSÉ AUX MINISTRES DU CULTE}

S'agissant des relations entre une organisation religieuse et ses personnels, la Cour EDH a dégagé à de nombreuses reprises une obligation de loyauté spécifique de ces derniers (2.1) qui s'inscrit dans la protection de l'autonomie organisationnelle et doctrinale de l'organisation. Ce devoir de loyauté entraîne par ricochet une limitation des droits substantiels du ministre du culte, particulièrement en matière de liberté individuelle de religion (2.2) et de droit au respect de la vie privée et familiale (2.3).

\subsection{UN DEVOIR DE LOYAUTÉ LIÉ À LA MISSION SPÉCIFIQUE DES MINISTRES DU CULTE}

Dans l'affaire Sindicatul «Păstorul cel Bun» ${ }^{29}$, la Grande Chambre souligne, à propos des prêtres de l'Église orthodoxe roumaine, que «le travail des membres du clergé présente la particularité de poursuivre une finalité spirituelle et d'être accompli dans le cadre d'une Église pouvant prétendre à un certain degré d'autonomie. Il en résulte que les obligations des membres du clergé sont d'une nature particulière en ce que ceux-ci sont soumis à un devoir de loyauté accru, lui-même fondé sur un engagement personnel de chacun de ses membres qui est censé être définitif» (§ 144).

Bien entendu, tous les personnels ne sont pas tenus au même degré de loyauté et «[...] la mission spécifique confiée à l'intéressé dans le cadre d'une organisation religieuse est un aspect à prendre en considération pour déterminer si cette personne doit être soumise à une obligation de loyauté

29. CEDH, Gde ch., 9 juill. 2013, n²330/09, Sindicatul «Păstorul cel Bun» c. Roumanie. 
accrue $^{30}$ ». À plusieurs reprises, la Cour a ainsi rappelé que dans l'appréciation de la gravité du comportement d'une personne employée au service de l'Église, il y a lieu de tenir compte de la «proximité» de son activité avec «la mission de proclamation de l'Église ${ }^{31} »$. Ce vocabulaire emprunté à l'article 5 du règlement fondamental de l'Église catholique allemande pour le service ecclésial ${ }^{32}$ rend bien sûr compte du rôle et de la mission de l'Église et des personnes qui y concourent en contexte chrétien. Cette estimation de l'apport particulier de certains personnels à la transmission du message et des rites religieux - estimation qui reste confiée à l'organisation religieuse - est certainement transposable à d'autres cultes en fonction de leur autocompréhension. On peut en tout cas affirmer que parmi les différents personnels, le «ministre du culte» tel qu'il peut être défini au sein de chaque communauté religieuse participe au premier titre à sa mission spirituelle et qu'il est donc soumis à un devoir de loyauté accru envers l'organisation concernée et la doctrine qu'elle professe.

La Cour lie souvent cette obligation de loyauté à la nécessité de préserver la «crédibilité » de l'organisation religieuse à l'égard du public, y compris dans des cas où le comportement à l'origine du licenciement relève de la sphère privée et familiale, et même "en l'absence de médiatisation de l'affaire ou de répercussions publiques importantes du comportement en question ${ }^{33}$ ». Il s'agit en quelque sorte d'affirmer par l'exemple le caractère obligatoire des prescriptions morales et des règles de comportement auxquelles les fidèles et a fortiori les personnels d'une Église ou d'une communauté religieuse doivent se soumettre ${ }^{34}$, et en tout premier lieu les ministres dans le cadre de leurs fonctions tout comme dans des situations extraprofessionnelles.

\subsection{PAS DE LIBERTÉ DE RELIGION POUR LES MINISTRES DU CULTE}

Dans une décision déjà ancienne concernant le licenciement d'un pasteur de l'Église nationale danoise - lequel arguait de sa liberté de religion pour

30. CEDH, Gde ch., 12 juin 2014, n 56030/07, Fernández Martínez c. Espagne, § 131.

31. Ibid., § 140. V. aussi CEDH, 23 sept. 2010, n 1620/03, Schüth c. Allemagne, § 69.

32. Grundordnung der Katholischen Kirche für den kirchlichen Dienst im Rahmen kirchlicher Arbeitsverhältnisse. Art. 5 - Manquements aux obligations de loyauté, cité dans Schüth c. Allemagne, \& 38.

33. CEDH, 23 sept. 2010, $\mathrm{n}^{\circ}$ 425/03, Obst c. Allemagne, § 51; V. aussi à la même date $\mathrm{n}^{\circ}$ 1620/03, Schüth c. Allemagne, §§ 63 et 66; 3 févr. 2011, $\mathrm{n}^{\circ} 18136 / 02$, Siebenhaar c. Allemagne, § 46; Gde ch., 12 juin 2014, n 56030/07, Fernández Martinez c. Espagne, $\S \S 137$ et 146 .

34. V. CEDH, 10 juin 2010, n 302/02, Témoins de Jéhovah de Moscou et autres c. Russie, $\$ 118$. 
subordonner le baptême des enfants à la condition que leurs parents suivent des leçons d'enseignement religieux -, la Commission européenne des droits de l'homme a clairement énoncé que «[La] liberté personnelle de pensée, de conscience et de religion [des ecclésiastiques] s'exerce au moment d'accepter ou de refuser une fonction ecclésiastique et, au cas où ils viendraient à être en désaccord avec les enseignements de l'Église, leur droit de quitter celle-ci sauvegarde leur liberté de religion. [...] En d'autres termes, [...] les Églises ne sont pas tenues d'assurer la liberté de religion de leurs prêtres et de leurs fidèles ${ }^{35} »$. La Cour EDH a ainsi estimé à plusieurs reprises qu'en cas de désaccord doctrinal entre un ministre et l'organisation qui l'emploie, la liberté de religion de celui-ci s'exerce uniquement par sa faculté de quitter librement la communauté ${ }^{36}$. Elle a maintenu cette approche en considérant plus largement que l'article 9 de la Convention ne garantit aucun droit individuel à la dissidence à l'intérieur d'un organisme religieux: l'autonomie des communautés religieuses implique l'acceptation par l'État de leur droit à réagir conformément à leurs propres règles et intérêts aux mouvements qui surgiraient en leur sein «et qui pourraient présenter un danger pour leur cohésion, pour leur image ou pour leur unité $e^{37} »$.

Dans des affaires récentes ${ }^{38}$ relatives à des employeurs restreignant la faculté d'un employé à se livrer à des pratiques religieuses, la Cour a fait évoluer sa jurisprudence en privilégiant la mise en balance des intérêts en cause plutôt que de conclure, comme elle l'avait fait précédemment, à l'absence d'ingérence dans l'exercice de la liberté de religion du requérant au motif qu'il pouvait démissionner de ses fonctions ${ }^{39}$. On peut présager cependant que cette dynamique ne concernera pas les ministres du culte, considérés comme les principaux garants du respect de la doctrine au sein de leur confession. La liberté de religion est d'abord celle de l'Église ou de la communauté religieuse qui impose à ses ministres les règles liées à son identité. La dimension collective de la liberté de religion - l'autonomie des communautés religieuses - l'emporte donc sur la dimension individuelle de

35. Comm. EDH, déc. 8 mars 1976, n 7374/76, X. c. Danemark; V. aussi Comm. EDH, 8 mars $1985, \mathrm{n}^{\circ} 11045 / 84$ Knudsen $c$. Norvège.

36. V. par ex. CEDH, 15 sept. 2009, n 798/05, Mirolubovs et autres c. Lettonie, § 80; Gde ch., 9 juill. 2013, n 2330/09, Sindicatul «Păstorul cel Bun» c. Roumanie, § 137.

37. CEDH, Gde ch., 9 juill. 2013, n 2330/09, Sindicatul «Păstorul cel Bun» c. Roumanie, § 165. V. aussi CEDH, Gde ch., 26 oct. 2000, n 30985/96, Hassan et Tchaouch c. Bulgarie, \& 78.

38. CEDH, 15 janv. 2013, $\mathrm{n}^{\text {os }} 48420 / 10,59842 / 10,51671 / 10$ et 36516/10, Eweida et a. c. Royaume-Uni, \& 83.

39. Comm. EDH, déc. 3 déc. 1996, n 24949/94, Konttinen c. Finlande; déc. 9 avr. 1997 , $\mathrm{n}^{\circ}$ 29107/95, Stedman c. Royaume-Uni. 
cette même liberté appliquée aux ministres du culte. Il en va de même pour d'autres droits substantiels des ministres qui peuvent paraître cependant davantage déconnectés de leurs fonctions, comme leur droit au respect de la vie privée et familiale.

\subsection{UN DROIT AU RESPECT DE LA VIE PRIVÉE ET FAMILIALE LIMITÉ}

La Cour EDH a eu l'occasion de se prononcer à plusieurs reprises sur des sanctions prises à l'égard de personnels laïcs exerçant dans des organisations confessionnelles en raison d'un comportement relevant de la sphère privée. Dans les affaires Obst et Schüth relatives à la contestation de licenciements pour adultère, la Cour a ainsi examiné la mise en balance effectuée par les juridictions du travail entre les droits des employés et ceux de l'Église employeur ainsi que la question de la proximité des activités des requérants (respectivement directeur des relations publiques dans l'Église mormone et organiste d'une paroisse catholique) avec la mission de proclamation de l'Église. La Cour conclut dans l'affaire Schüth à la violation du droit du requérant au respect de sa vie privée et familiale (art. $8 \mathrm{CEDH}$ ) et à l'absence de violation dans l'affaire $\mathrm{Obst}^{40}$. Ces décisions qui mettaient notamment l'accent sur la nécessité d'un contrôle juridictionnel conforme à la Convention furent remarquées par la doctrine ${ }^{41}$. Elles sont d'ailleurs mentionnées dans l'arrêt de Grande Chambre Fernández Martínez ${ }^{42}$ par lequel la Cour EDH se prononce pour la première fois sur le licenciement d'un prêtre, marié et père de famille. Prêtre et professeur de religion catholique en Espagne, M. Fernández avait sollicité après son mariage une dispense de célibat qu'il a obtenu treize années plus tard, après être apparu avec femme et enfants dans la presse en manifestant des opinions allant à l'encontre de certaines positions de l'Église sur des questions de vie sexuelle et familiale. La perte de son état clérical s'accompagne alors du non-renouvellement de son contrat de professeur dans un lycée public, à la suite d'une demande en ce sens de l'évêque au ministère de l'Éducation.

40. CEDH, 23 sept. 2010, n 1620/03, Schüth c. Allemagne; n 425/03, Obst c. Allemagne.

41. D. 2011, p. 1637, chron. J.-P. Marguénaud et J. Mouly; D. 2012, p. 901, obs. P. LokieC et J. Porta; RDT 2011, p. 5, obs. J. Coundr; JCP G 2010, act. 1006, obs. M. LÉvinet.

42. Dr. soc. 2015, p. 719, étude J.-P. Marguénaud et J. Mouly; JCP 2014, act. 845, obs. G. Gonzalez; AJDA 2014, p. 1763, obs. L. Burgorgue-Larsen; G. Gonzalez, «Consolidation du principe d'autonomie des Églises en droit européen des droits de l'homme», art. cit. 
La Cour considère que le non-renouvellement du contrat du requérant en raison d'événements principalement liés à des choix personnels a gravement compromis ses possibilités d'exercer son activité professionnelle, mais que cette ingérence dans l'exercice de son droit au respect de sa vie privée n'est pas disproportionnée. En effet, «les juridictions nationales ont procédé à une mise en balance circonstanciée et approfondie des intérêts en jeu, [...] dans les limites que leur imposait le respect dû à l'autonomie de l'Église catholique» (\$151), le requérant s'étant «lui-même placé, sciemment, dans une situation totalement contraire aux préceptes de l'Église» (§ 146) alors que la proximité entre son activité professionnelle et la mission de proclamation de l'Église "était manifestement grande » (§ 140). Pour être "crédible », l'enseignement de la religion doit être donné par une personne « dont le mode de vie et les déclarations publiques ne sont pas en contradiction flagrante avec la religion en question, dès lors surtout que celle-ci prétend régir la vie privée et les convictions personnelles de ses adeptes» (§ 138). La Cour estime que le requérant, en signant ses contrats d'emploi successifs, a accepté en connaissance de cause et volontairement un devoir de loyauté accru, lequel n'a donc pas été rempli, «ce qui a limité dans une certaine mesure l'étendue de son droit au respect de sa vie privée et familiale» (§ 135).

Il est à noter que dans cette affaire le requérant est employé et rémunéré comme enseignant par l'État et que son licenciement a donc été décidé par une autorité publique, même s'il résulte d'une "proposition contraignante» émanant d'une autorité ecclésiastique. Selon la Cour, cet aspect n'est toutefois pas de nature à influer sur les mesures que l'Église peut adopter en cas de manquement à l'obligation de loyauté, car celle-ci a un rôle exclusif dans la désignation et le licenciement des professeurs de religion (§ 143).

La Grande Chambre a suivi un raisonnement identique et abouti à la même conclusion de non-violation de l'article 8 dans une affaire concernant cette fois un enseignant laï de religion catholique en Croatie, révoqué après le retrait de son investiture canonique à la suite de son divorce et de son remariage civil ${ }^{43}$. Dans le cas d'espèce, le fait que son mode de vie contraire aux préceptes de l'Église n'ait pas fait l'objet de publicité ne constitue pas, selon la Cour, un élément décisif dans l'appréciation des conséquences emportées par la décision de révocation (§ 99). Le requérant avait conscience de l’importance du sacrement du mariage pour l'Église et il s'est mis lui-même dans une situation ayant abouti à la perte de son mandat canonique en décidant 
«de ne pas tenir compte des exigences d'allégeance spéciale à l'égard des enseignements et de la doctrine de l'Église» (§ 96).

La Cour précise certes dans ces deux affaires que les juridictions étatiques doivent vérifier que l'ingérence de l'employeur dans le droit au respect de la vie privée ne va pas au-delà de ce qui est nécessaire et qu'elle ne sert pas un but étranger à l'exercice de l'autonomie de la communauté religieuse. La variété des modèles de relations États-religions dans les pays européens la conduit cependant à accorder une large marge d'appréciation nationale en matière d'affaires religieuses et elle suit ici les juridictions nationales en admettant que l'ingérence incriminée était prévisible et a des bases légales internes, respectivement les dispositions des accords passés avec le Saint-Siège par l'Espagne (1979) et la Croatie (1996). La Cour n'a pas procédé elle-même à une réelle mise en balance des intérêts en jeu à l'aune du principe de proportionnalité qui permettrait de garantir une protection effective des droits fondamentaux des requérants ${ }^{44}$.

Les obligations de loyauté spécifiques imposées à leurs employés par les organisations religieuses sont particulièrement renforcées s'agissant des ministres du culte. Considérés comme des éléments essentiels de la vie de leur communauté, ils sont aussi les principaux vecteurs de l'image véhiculée auprès des fidèles et du public. Leurs droits substantiels demeurent alors largement subordonnés au droit d'autonomie des organisations dont les effets peuvent paraître dans certains cas disproportionnés, la Cour EDH respectant en la matière la marge d'appréciation des États pour lesquels les ministres du culte ne sont pas des professionnels comme les autres.

44. V. en ce sens l'opinion dissidente commune à huit juges dans la décision de Grande Chambre Fernández Martínez. 